\title{
Serratia marcescens as Opportunistic Pathogen and the Importance of Continuous Monitoring of Nosocomial Infection in Makah City, Saudi Arabia
}

\author{
Hani S. Faidah1, Sami S. Ashgar1, Abeer A. A. Barhameen1, Hamdi M. El-Said1*, \\ Abdelrahman Elsawy ${ }^{2,3}$ \\ ${ }^{1}$ Medical Microbiology Department, Faculty of Medicine, Umm Al-Qura University, Makah City, Saudi Arabia \\ ${ }^{2}$ Al-Noor Specialist Hospital, Makah City, Saudi Arabia \\ ${ }^{3}$ Department of Medical Microbiology, Faculty of Medicine, Al-Azhar University, Cairo, Egypt \\ Email: ${ }^{*}$ hamdimustafa1@gmail.com
}

Received 27 May 2015; accepted 10 August 2015; published 13 August 2015

Copyright (C) 2015 by authors and Scientific Research Publishing Inc.

This work is licensed under the Creative Commons Attribution International License (CC BY).

http://creativecommons.org/licenses/by/4.0/

(c) (i) Open Access

\section{Abstract}

The aim of this study was to the evaluation of frequency and distribution of Serratia marcescens in the hospital departments and determination of antimicrobial resistance of the isolated strains. Methods: The study included 81 Serratia marcescens strains isolated from 61 patients hospitalized in the in the different hospital wards of Al-Noor Specialist Hospital within the period from 1/11/ 2012 to $1 / 11 / 2013$. The strains were isolated from wound swabs, blood cultures, sputum, urine culture, fluid, catheter and throat swab, wound swabs, blood cultures and cerebrospinal fluid. Results: The isolates were identified by conventional method and the results and susceptibility testing were confirmed by VITEC-2 Compact. Most frequently Serratia marcescens has been implicated in ICU [21\%] followed by male medical [18.5\%] and emergency department [12.3\%]. The resistance of Serratia strains was high, excepting imipenem (15\%), Meropenem (27) and the resistance was higher with ampicillin $(97.5 \%)$, Cefoxitin $(90 \%)$ and Tetracycline $(86 \%)$. Conclusion: Continuous monitoring of nosocomial infections is indispensable. Phenotypic characterization of the isolates is useful for studying the relationship of microbial pathogens.

\section{Keywords}

Serratia marcescens, Antimicrobial Resistance, Nosocomial İnfection

\footnotetext{
${ }^{*}$ Corresponding author.
}

How to cite this paper: Faidah, H.S., Ashgar, S.S., Barhameen, A.A.A., El-Said, H.M. and Elsawy, A. (2015) Serratia marcescens as Opportunistic Pathogen and the Importance of Continuous Monitoring of Nosocomial Infection in Makah City, Saudi Arabia. Open Journal of Medical Microbiology, 5, 107-112. http://dx.doi.org/10.4236/ojmm.2015.53013 


\section{Introduction}

Serratia marcescens is opportunistic pathogen causing nosocomial infection. The organism has been implicated abroad range of infections including respiratory tract, bloodstream, central nervous system, urinary tract and epiglottitis [1] [2].

Patients most at risk are those in intensive care units who are subjected to medical devices, especially central venous catheters, and those treated with broad-spectrum antimicrobial drugs [3]. The first description of nosocomial infection caused by Serratia marcescens was Wheat's report of 11 cases over a 6-month period in 1951 at Stanford University Hospital [4]. Infections caused by this organism have been reported with increasing frequency since 1960 [5]. Serratia marcescens has expressed the ability to survive and grow under extreme conditions, including in disinfectants, antiseptics and double-distilled water [6]. Serratia can be easily spread in the nosocomial environment and contaminate medical equipment, instruments, commercial fluids, disinfectants and dispensers [1]. Poor adherence to hand hygiene of healthcare staff is also implicated in infections and outbreaks [7]. Multidrug resistance of serratia strains poses another serious threat for patient, restricting therapeutic options.

Serratia spp. constitutively possesses chromosomally encoded, inducible AmpC-lactamases, and may acquire plasmid-mediated extended-spectrum-lactamases (ESBLs). Therefore, they have the ability to develop resistance to many lactam antibiotics [8] [9]. Patients with S. marcescens infections often receive empirical antimicrobial agents before information about antimicrobial susceptibility becomes available. The empirical antimicrobial agents used often include extended-spectrum-lactam drugs, such as third- and fourth-generation cephalosporin's, and carbapenems. Several studies have demonstrated that multidrug-resistant strains of S. marcescens can cause serious nosocomial infections [10] [11]. In the current study we analyzed the distribution of Serratia marcescens strains in the Al-Noor Specialist Hospital in the western part of Saudi Arabia, and evaluated the risk factors of outbreak of Serratia marcescens and their resistance to the antibiotics, and the importance of continuous monitoring of nosocomial infection.

\section{Methods}

The study was conducted at a Al-Noor Specialist Hospital in the western part of Saudi Arabia. Retrospectively, the medical records of patients who were diagnosed with Serratia marcescens infection were reviewed and data were extracted. The analysis included all isolates of Serratia marcescens from hospitalized patients Involving 61 patients, in the different wards of the Hospital involved in this study. Serratia marcescens strains in our study were isolated from various clinical specimens, wound swabs (26), blood cultures (26), fluid (3), sputum (15), urine culture (7), catheter (2), and throat swab (2) within the period from 1/11/2012 to 1/11/2013. The microbiology laboratory had performed identification of Serratia marcescens based on conventional biochemical testing according to the CLSI (Clinical Laboratory Standard Institute) [12] and the species confirmation were conducted by Microscan for automated bacterial identification testing system. And the interpretative criteria established by the Clinical Laboratory Standards Institute (CLSI) guidelines [13]. Antimicrobial susceptibility testing was performed by Microscan for automated bacterial susceptibility testing system, bacterial inoculated were prepared by suspending the freshly grown bacteria in normal sterile saline adjusted to a 0.5 McFarland standard. Antimicrobial susceptibility was determined for ampicillin, Cefepime, Ceftazidime, Piperacillin/Tazobactam, Tetracycline, Gentamycin, Amikacin, Cefoxitin, Cefotaxime, Imipenem, Ciprofloxacin, Meropenem, Trimethoprim/ Sulfamethoxazole, Tigecycline, Moxifloxacin, Fosfomycin and Ertapenem. The statistical data were analyzed by descriptive statistical values (statistical measures of counting-frequencies, percentages).

\section{Results}

A total of 81 Serratia marcescens strains were isolates from 61 patients hospitalized in the different hospital wards. Serratia marcescens infections were registered in ICU [21\%] followed by male medical [18.5\%], emergency department [12.3\%], female medical [7.4\%], Critical Care Unit (CCU) [6.2\%], Diabetic Foot Dressing [6.2\%], Orthopedics [6.2\%], E.N.T. Unit [6.2\%], Urology [4.9\%], Surgery [3.7\%], Plastic Surgery [3.7\%], Pediatric medical [2.4\%], Hemodialysis [2.4\%], and neurosurgery [1.2\%] (Table 1).

Serratia marcescens strains in our study were isolated from various clinical specimens, wound swabs [32\%], blood cultures [32\%], sputum [18.5], urine culture [8.6\%], fluid [3.7], catheter [2.5\%] and throat swab [2.5\%] (Table 2). 
Table 1. Illustrates the distribution of the Serratia marcescens isolated in different hospital wards compared with the number of patients.

\begin{tabular}{cccc}
\hline Hospital department & Number of strains & \% & Number of Patient \\
\hline CCU & 5 & 6.2 & 2 \\
Diabetic Foot Dressing & 5 & 6.2 & 3 \\
Female Medical (A) & 5 & 6.2 & 5 \\
Female Medical (B) & 1 & 1.2 & 2 \\
Female Plastic Surgery & 2 & 2.4 & 2 \\
Female Surgery & 2 & 2.4 & 1 \\
Hemodialysis (A.K.C.) & 2 & 2.4 & 13 \\
ICU & 17 & 21 & 1 \\
Male Ent & 4 & 4.9 & 13 \\
Male Medical (B) & 15 & 18.5 & 1 \\
Male Neurosurgery & 1 & 1.2 & 9 \\
Emergency department & 10 & 12.3 & 1 \\
Male Plastic Surgery & 1 & 1.2 & 1 \\
Male Surgical & 1 & 1.2 & 2 \\
Orthopedics & 5 & 6.2 & $\mathbf{6 1}$ \\
Pediatric Medical & 2 & 2.4 & 2 \\
Urology & 3 & $\mathbf{1 0 0}$ & 2.7 \\
Total & 51 & & 2 \\
\hline & 5 & 2.4 \\
\hline
\end{tabular}

Table 2. Illustrates the localizations in the body of the infections produced by Serratia marcescens.

\begin{tabular}{ccc}
\hline Clinical specimens & No. S. marcescens isolates & \% \\
\hline Fluid, Other Body & 3 & 3.7 \\
Sputum & 15 & 18.5 \\
Blood & 26 & 32 \\
Tip, Catheter & 2 & 2.5 \\
Wound & 26 & 32 \\
Throat Swab & 2 & 2.5 \\
Urine Culture & 7 & 8.6 \\
Total & $\mathbf{8 1}$ & $\mathbf{1 0 0}$ \\
\hline
\end{tabular}

The strains were identified with current phenotypic methods and antibiotic susceptibility patterns. The isolated showed resistance of, ampicillin (97.5\%), Cefoxitin (90\%), Tetracycline (86\%), Piperacillin/Tazobactam (63\%), Moxifloxacin (60\%), Ceftazidime (59\%), Fosfomycin (58\%) and Tigecycline (53\%). Isolates remained susceptible to, Imipenem (85\%), Meropenem (73\%), Gentamycin (69\%), Amikacin (67\%), Cefepime (67\%), Trimethoprim/Sulfamethoxazole (59\%), Ertapenem (57\%), Ciprofloxacin (51\%) and Cefotaxime (41\%) (Table 3).

The most active antibiotic being Imipenem and Meropenem. Resistance to other tested antibiotics was variable with significantly higher resistance to ampicillin, cefoxitin and Tetracycline. The most common infections caused by Serratia marcescens the hospital were wound and blood stream infections (64\%), followed by pneumonia (18.5\%). 
Table 3. Illustrates the percentages of the resistant of 81 Serratia marcescens strains for each of the tested antibiotics..

\begin{tabular}{ccccc}
\hline Antimicrobial agent & $\begin{array}{c}\text { Number of } \\
\text { susceptible isolates }\end{array}$ & $\begin{array}{c}\text { Susceptibility } \\
\text { rate (\%) }\end{array}$ & $\begin{array}{c}\text { Number of resistant } \\
\text { isolates }\end{array}$ & $\begin{array}{c}\text { Resistant } \\
\text { rate (\%) }\end{array}$ \\
\hline Imipenem & 69 & 85.2 & 12 & 14.8 \\
Meropenem & 59 & 73 & 22 & 27 \\
Gentamycin & 56 & 69 & 25 & 31 \\
Amikacin & 54 & 67 & 27 & 33 \\
Cefepime & 54 & 67 & 27 & 33 \\
Trimethoprim/Sulfamethoxazole & 48 & 59 & 33 & 41 \\
Ertapenem & 46 & 57 & 35 & 49 \\
Ciprofloxacin & 41 & 51 & 40 & 49 \\
Cefotaxime & 41 & 51 & 40 & 53.1 \\
Tigecycline & 41 & 46.9 & 43 & 58 \\
Fosfomycin & 41 & 42 & 47 & 59 \\
Ceftazidime & 33 & 41 & 48 & 60.5 \\
Moxifloxacin & 32 & 39.5 & 49 & 63 \\
Piperacillin/Tazobactam & 30 & 37 & 51 & 96.5 \\
Tetracycline & 11 & 13.5 & 60 & 93.1 \\
Cefoxitin & 8 & 9.9 & 79 & \\
Ampicillin & 2 & 2.5 & & 43 \\
\hline
\end{tabular}

\section{Discussion}

In our study, 81 Serratia marcescens were isolated. During the study from 61 patients hospitalized in the different hospital wards. There have been initially analyzed the frequency of isolation of the Serratia marcescens strains from various biological products from patients hospitalized in 2013. Serratia marcescens more frequently etiological involved in blood stream infections, wounds infections, followed by the infections of lower respiratory tract and urinary tract infections. Rarely, Serratia marcescens was isolated from peritoneal, pleural and joint fluids. Previous studies demonstrated variable distribution of Serratia marcescens in different hospital wards. Over the last 40 years, Serratia marcescens has become important cause of nosocomial infections. There have been many reports concerning the identification, antibiotic susceptibility, pathogenicity and epidemiological investigation of this microorganismism [14]-[16]. Outbreaks have been described in a variety of clinical settings and different environmental sources have been identified as reservoirs. Several studies described infections caused by Serratia marcescens associated with contaminated disinfectants and antiseptics [17]. Bosi et al. reported outbreak of Serratia marcescens infection in the neurosurgery intensive care unit due to contamination of hexetidine solutions [18]. Patients with Serratia marcescens respiratory tract infections may become colonized with Serratia marcescens after instrumentation, reported outbreaks being associated with contaminated bronchoscopes [19]. Serratia marcescens is able to colonize the hospital environment and equipment, having a predilection for growth in moist areas. Soaps, distilled water, sponges, irrigating fluids and hand lotions have been implicated as a source in previous reports [20]. The antibiotic susceptibility pattern observed in our study is in accordance with some previous studies. All Serratia marcescens isolates in the current study were susceptible to Imipenem and Meropenem to high degree. Resistance to Gentamycin, Amikacin, trimethoprim/sulfamethoxazole, Cefepime, Ertapenem, Ciprofloxacin, Cefotaxime and Ceftazidime were variable degrees. The resistance was higher with Ampicillin, Cefoxitin and Tetracycline. The wide distribution of Serratia strains in different hospital wards illustrated by this study as also the various localizations of the infections produced by these strains are in accordance with other published results of similar studies [21]-[24]. Like in the case of other multidisciplinary hospitals, there have been found different levels of resistance to various antibiotics, the most active 
antibiotic being imipenem [25]-[27]. Serratia infections may be difficult to treat as they may exhibit multidrug resistance. Spread of these resistant strains poses a significant threat among hospitalized patients. Nosocomial infections with multidrug resistant organisms (MDROs) are a major cause of morbidity and mortality. A lot of patients were colonized with MDROs on admission, indicating the importance of screening on admission to intensive care units (ICUs). Screening on admission allows early detection and limits dissemination of these strains with application of appropriate control measures. The factors which predispose colonization and infection with MDROs are most often transmitted among patients by the hands of personnel. So, it was suggested that permanent control of personnel is necessary [28]. Our findings were identical with some results on the studies in the world and different with others. But the resistance of bacteria to antibiotics rises in all the studies that have been made. Making it an important source of infection in hospitals must be addressed and control for not increasing the spread of these infections.

\section{Conclosions}

1) Serratia marcescens could be isolated from diverse wards, especially from Intensive Care Unit [21\%] followed by male medical [18.5\%] and emergency department [12.3\%].

2) The resistance of Serratia marcescens to antibiotics was high, excepting imipenem [14\%] that proved to be a reserve antimicrobial for the infections with these strains. For aminoglycosides, the resistance was in the case of gentamicin [31\%] but there were also been found strains resistant to amikacin [33\%]. For all antimicrobials currently used in the treatment of urinary infections the percentages of resistant Serratia marcescens were high.

3) The obtained results indicate the importance of identifying Serratia marcescens as a significant agent of nosocomial infections and of the detection of multi-drug-resistant strains that could cause therapeutic difficulties.

4) Early implementation of infection prevention and control measures, including adequate decontamination of chest tube drainage devices between each patient, controlled the extent of the outbreak promptly.

5) Strict adherence to appropriate infection prevention and control standards is essential to avoid transmission of this organism in the healthcare environment.

\section{References}

[1] Musham, C.K., Jarathi, A. and Agarwal, A. (2012) Acute Epiglottitis Due to Serratia marcescens in an Immune Competent Adult. The American Journal of the Medical Sciences, 344, 153-154.

[2] Polilli, E., Parruti, G., Fazii, P., et al. (2011) Rapidly Controlled Outbreak of Serratia marcescens Infection/Colonization in a Neonatal Intensive Care Unit, Pescara General Hospital, Pescara, April 2011. Euro Surveill, 16.

[3] Hejazi, A. and Falkiner, F.R. (1997) Serratia marcescens. Journal of Medical Microbiology, 46, 903-912. http://dx.doi.org/10.1099/00222615-46-11-903

[4] Wheat, R.P., Zuckerman, A. and Rantz, L.A. (1951) Infection Due to Chromobacteria: Report of Eleven Cases. JAMA Internal Medicine, 88, 461-466. http://dx.doi.org/10.1001/archinte.1951.03810100045004

[5] Dodson, W.H. (1968) Serratia marcescens Septicaemia. JAMA Internal Medicine, 121, 145-150. http://dx.doi.org/10.1001/archinte.1968.03640020033006

[6] Sautter, R.L., Mattman, K.H. and Legaspi, R.C. (1984) Serratia marcescens Meningitis Associated with a Contaminated Benzalkonium Chloride Solution. Infection Control, 5, 223-225.

[7] Buffet-Bataillon, S., Rabier, V., Betremieux, P., Beuchee, A., Bauer, M., Pladys, P., et al. (2009) Outbreak of Serratia marcescens in a Neonatal Intensive Care Unit: Contaminated Unmediated Liquid Soap and Risk Factors. Journal of Hospital Infection, 72, 17-22. http://dx.doi.org/10.1016/j.jhin.2009.01.010

[8] De Vries, J.J., Baas, W.H., van der Ploeg, K., Heesink, A., Degener, J.E. and Arends, J.P. (2006) Outbreak of Serratia marcescens Colonization and Infection Traced to a Healthcare Worker with Long-Term Carriage on the Hands. Infection Control and Hospital Epidemiology, 27, 1153-1158.

[9] Bennett, P.M. and Chopral. I. (1993) Molecular Basis of Beta-Lactamase Induction in Bacteria. Antimicrobial Agents and Chemotherapy, 37, 153-158. http://dx.doi.org/10.1128/AAC.37.2.153

[10] Wul, T., Tsou, M.F., Wu, H.J., Chen, H.E., Chuang, Y.C. and Yu, W.L. (2004) Survey of CTX-3 Extended-Spectrum Beta-Lactamase (ESBL) among Cefotaxime-Resistant Serratia marcescens at a Medical Center in Middle Taiwan. Diagnostic Microbiology and Infectious Disease, 49, 125-129. http://dx.doi.org/10.1016/j.diagmicrobio.2004.02.004

[11] Henjyoji, E.Y., Whitson, T.C., Oashi, D.K. and Allen, B.D. (1971) Bacteremia Due to Serratia marcescens. The Jour- 
nal of Trauma, 11, 417-421. http://dx.doi.org/10.1097/00005373-197105000-00006

[12] Yu, W.L., Lin, C.W. and Wang, D.Y. (1998) Serratia marcescens Bacteremia: Clinical Features and Antimicrobial Susceptible of the Isolates. Journal of Microbiology, Immunology, and Infection, 31, 171-179.

[13] Jorgansen, H.J. (2011) Antibacterial Agents and Susceptibility Test Methods. In: Versolovic, J., Caroll, K.C., Funke, G., Jorgansen, H.J., Landry, M.L and Warnock, D.W., Eds., Manual of Clinical Microbiology, 10th Edition, Vol. 1, American Society for Microbiology, Texas.

[14] Clinical and Laboratory Standards Institute (2005) Performance Standards for Antimicrobial Susceptibility Testing. CLSI, Wayne, CLSI Document M100-S15.

[15] Hejazi, A. and Falkiner, F.R. (1997) Serratia marcescens. Journal of Medical Microbiology, 46, 903-912. http://dx.doi.org/10.1099/00222615-46-11-903

[16] Sleigh, J.D. (1983) Antibiotic Resistance in Serratia marcescens. British Medical Journal, 287, 1651-1653. http://dx.doi.org/10.1136/bmj.287.6406.1651

[17] Knowles, S., Herra, C., Devitt, E., O’Brien, A., Mulvi-hill, E., McCann, S.R., et al. (2000) An Outbreak of Multi-Ply Resistant Serratia marcescens: The Importance of Persistent Carriage. Bone Marrow Transplantation, 25, 873-877. http://dx.doi.org/10.1038/sj.bmt.1702218

[18] Bosi, C., Davin-Regli, A., Charrel, R., Rocca, B., Mon-net, D. and Bollet, C. (1996) Serratia marcescens Nosocomial Outbreak Due to Contamination of Hexetidine Solution. Journal of Hospital Infection, 33, 217-224. http://dx.doi.org/10.1016/S0195-6701(96)90005-5

[19] Vandenbroucke-Grauls, C.M., Baars, A.C., Visser, M.R., Hulstaert, P.F. and Verhoef, J. (1993) An Outbreak of Serratia marcescens Traced to a Contaminated Bronchoscope. Journal of Hospital Infection, 23, 263-270.

[20] Miranda, G., Kelly, C., Solorzano, F., Leanos, B., Coria, R. and Patterson, J.E. (1996) Use of Pulsed-Field Gel Electrophoresis Typing to Study an Outbreak of Infection Due to Serratia marcescens in a Neonatal Intensive Care Unit. Journal of Clinical Microbiology, 34, 3138-3141.

[21] Choi, S.H., Lee, J.E., et al. (2007) Prevalence, Microbiology, and Clinical Characteristics of Extended-Spectrum Beta-Lactamase-Producing Enterobacter spp., Serratia marcescens, Citrobacter freundii and Morganella morganii in Korea. European Journal of Clinical Microbiology \& Infectious Diseases, 26, 557-561. http://dx.doi.org/10.1007/s10096-007-0308-2

[22] Dubouix, A., Roques, C., Segonds, C., et al. (2005) Epidemiological Investigation of a Serratia liquefaciens Outbreak in a Neurosurgery Department. Journal of Hospital Infections, 60, 8-13. http://dx.doi.org/10.1016/j.jhin.2004.09.029

[23] Fleisch, F., Zimmermann-Baer, U., et al. (2002) Three Consecutive Outbreaks of Serratia marcescens a Neonatal Intensive Care Unit. Clinical Infections Diseases, 34, 767-773.

[24] Iosifidis, E., Farmaki, E., et al. (2012) Outbreak of Bloodstream Infections Because of Serratia marcescens in a Pediatric Department. American Journal of Infection Control, 40, 11-15. http://dx.doi.org/10.1016/j.ajic.2011.03.020

[25] Rajput, A., Shah, U., et al. (2009) Serratia-An Emerging Pathogen in Hospital Environment. Gujarat Medical Journal, 64, 70-71.

[26] Sethuraman, S., Arunachalam, A., et al. (2011) Antimicrobial Sensitivity Profile of Serratia marcescens Strains Isolated in Government General Hospital, Nagapattinam, Tamilnadu, India. International Journal of Preclinical and Pharmaceutical Research, 2, 7-11.

[27] Kumar, A. and Worobec, E. (2002) Fluoroquinolone Resistance of Serratia marcescens: Involvement of a Proton Gradient-Dependent Efflux Pump. Journal of Antimicrobial Chemotherapy, 50, 593-596.

[28] Emily, R.M. (2011) Sydnor and Trish M. Hospital Epidemiology and Infection Control in Acute-Care Settings. Clinical Microbiology Reviews, 24, 141-173. http://dx.doi.org/10.1128/CMR.00027-10 